\title{
Coronary microvascular dysfunction: Filling the research gaps with careful patient selection
}

\author{
Christopher Schumann, MD, ${ }^{\mathrm{a}, \mathrm{b}}$ and Jamieson $M$. Bourque, $M D, M H S^{\mathrm{a}, \mathrm{b}}$ \\ a Department of Medicine, Cardiovascular Imaging Center, University of Virginia Health System, \\ Charlottesville, VA \\ b Department of Radiology, University of Virginia Health System, Charlottesville, VA
}

Received Aug 30, 2018; accepted Aug 30, 2018

doi: 10.1007/s12350-018-1449-7

\section{See related article, pp. 1844-1852}

Although obstructive coronary artery disease (CAD) has long been considered the cause of myocardial ischemia, there is increasing recognition of a subclass of patients who present with evidence of ischemia and are found to have no significant epicardial CAD. These patients with ischemia and no obstructive coronary disease (INOCA) have been shown to have worse outcomes than patients without ischemia. ${ }^{1}$ Jespersen et al. examined more than 11,000 subjects who presented with chest pain and had normal coronaries or diffuse nonobstructive $\mathrm{CAD}$ on angiography. After controlling for other cardiac risk factors, patients with symptoms of ischemia and normal coronary arteries had a $52 \%$ increased risk of major adverse cardiac events, defined as cardiovascular death, stroke, heart failure, or myocardial infarction, compared with a reference population without ischemic heart disease or symptoms thereof. $^{2}$

The mechanisms by which INOCA occurs and leads to worse outcomes have not been fully elucidated, but possible mechanisms include epicardial endothelial dysfunction and coronary microvascular dysfunction (CMD). CMD includes both microvascular endothelial and nonendothelial dysfunction that results in decreased

Funding Christopher Schumann receives support from NIH-T32EB003841. Jamieson Bourque receives support from NIH K23HL119620-02.

Reprint requests: Jamieson M. Bourque, MD, MHS Department of Medicine Cardiovascular Imaging Center, University of Virginia Health System, 800662, 1215 Lee Street, Charlottesville, VA 22908; jamieson2@gmail.com

J Nucl Cardiol 2019;26:1853-6.

$1071-3581 / \$ 34.00$

Copyright (C) 2018 American Society of Nuclear Cardiology. myocardial perfusion. ${ }^{1}$ CMD can be assessed through evaluation of the myocardial flow reserve (MFR), which is also referred to as coronary flow reserve or myocardial perfusion reserve. MFR is defined as the ratio of coronary flow under maximal vasodilation to resting flow. In the absence of epicardial stenosis, the microvasculature primarily determines flow resistance. As a result, MFR is determined by the microvasculature in patients without obstructive epicardial CAD. ${ }^{3}$ Multiple studies have shown PET to be an accurate and reproducible technique for measuring myocardial blood flow (MBF) and MFR and has been used in multiple studies to examine the diagnostic and prognostic impact of this measurement. ${ }^{4-12}$ A MFR $<2.0$ has frequently been used as a threshold to diagnose CMD, but an exact cutoff remains unclear, as various studies have used MFR values ranging from 1.5 to 2.6 to diagnose $\mathrm{CMD} .^{13}$

Irrespective of the cutoff used, impaired MFR has been shown to predict worse outcomes. Brainin et al. performed a meta-analysis that included 10 studies that used PET to diagnose CMD. They found that CMD patients without obstructive CAD had an overall relative risk (RR) of major adverse cardiac events of 2.44 (95\% CI 1.80 to 3.30$).{ }^{14}$ In a subgroup analysis of the six studies in patients with CMD whose presentation was stable angina, they found an overall RR of MACE of 2.52 (95\% CI 1.85 to 3.43$).^{7-12}$ Figure 1 highlights two limitations of the studies included in this meta-analysis when used to assess patients with CMD and no obstructive epicardial CAD: (1) follow-up was only short term; (2) obstructive CAD was not specifically excluded through noninvasive or invasive angiography. In the largest study in this meta-analysis, Murthy et al. performed a seminal and robust analysis of $>1200$ subjects with no evidence of obstructive CAD on rest/ stress PET imaging. They found patients with CMD to have a significant increase in MACE (HR 0.8 per $10 \%$ improvement in MFR), which was defined as cardiac 


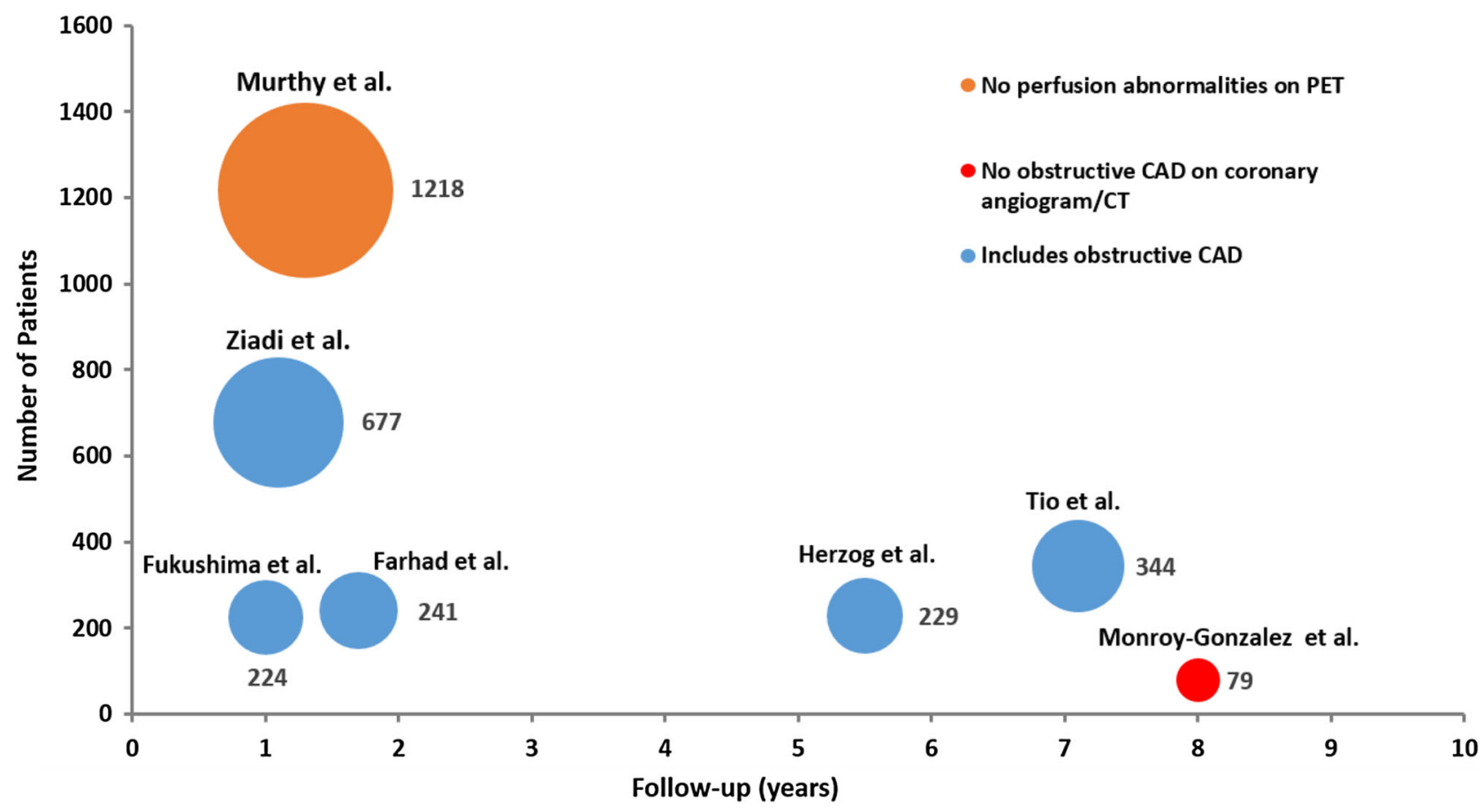

Figure 1. Comparison of number of patients, follow-up duration, and the presence of obstructive CAD in coronary microvascular dysfunction prognostic studies.

death, nonfatal MI, late revascularization, or CHF hospitalization. ${ }^{10}$ This study had a short-term follow-up horizon (mean of 1.1 years). In addition, while patients with perfusion abnormalities on rest/stress PET were excluded, the absence of obstructive CAD was not confirmed on invasive or CT coronary angiography. Thus, some patients with epicardial obstructive CAD may have been included. The other studies in the metaanalysis expressly included patients with obstructive epicardial CAD, resulting in a heterogenous study population.

As highlighted in Figure 1, there is a current gap in the literature assessing the prognostic impact of patients with chest pain and the confirmed absence of obstructive epicardial CAD on angiography over long-term followup. In this issue of the Journal, Monroy-Gonzalez et al. start filling in this research gap. They conducted a retrospective analysis of patients with the confirmed absence of obstructive epicardial CAD (no stenosis $\geq 30 \%$ on invasive or CT coronary angiography). The study population was composed of 79 patients who underwent PET myocardial perfusion imaging (MPI) with quantitation of MBF and MFR. They found 43\% prevalence of reduced MFR $<2.0$. Mortality and MACE (cardiac death, heart failure hospitalization, nonfatal MI, or late revascularization $\geq 90$ days) were assessed over a long-term follow-up median of 8 years (IQR 3 to 14 years). Unadjusted Kaplan-Meier analysis showed a significantly increased mortality risk and rate of MACE in subjects with $\mathrm{CMD}$ with a MFR $<2.0$. Univariable Cox regression analysis showed MFR to be a significant predictor of both mortality and MACE. Stress MBF only predicted MACE but not all-cause mortality. As illustrated in Figure 1, this study stands out from prior CMD studies by both reporting long-term data and by explicitly excluding patients with obstructive epicardial CAD identified on angiography.

Limitations of this study include a low sample size (79 patients) and high rate of incomplete follow-up (35/ $79,44.3 \%$ ) for MACE. Mortality data were available for all patients with the inherent limitations of government mortality databases. In addition, the absolute number of events in this study was low, with only 6 deaths and 6 MACE events; however, the relative event rate was adequate to produce a statistically significant result through univariable analysis. Multivariable analysis was unable to be performed due to the low number of events.

While Monroy-Gonzalez et al. add an important contribution to the CMD literature by looking at longterm outcomes in the absence of obstructive epicardial CAD that is confirmed, the next steps in CMD research should include larger prognostic studies evaluating longterm outcomes data in this population. In addition, there is a significant need for prospective trials to evaluate CMD treatments. Given the significant symptom morbidity and multiple studies that have demonstrated 
increased mortality and MACE rates in patients with CMD, the need for effective therapies is apparent. Unfortunately, there is little data to support specific therapies in CMD. A systematic review by our group (Marinescu et al.) illustrates the lack of data to support treatment strategies in patients with the confirmed CMD. ${ }^{15}$ In our review of the literature, we noted that treatment studies for patients with chest pain and no obstructive epicardial CAD often included a heterogenous patient population. Without specifically testing for CMD, these study populations included patients with other causes of chest pain such as noncardiac chest pain, pericardial pain, and coronary vasospasm. We identified only 8 studies that met our inclusion criteria of evaluating patients with angina, no obstructive epicardial CAD, and a MFR cutoff of $<2.5$ as assessed by robust noninvasive (PET or CMR) or invasive techniques. Studies involving ACE inhibitors, estrogen, and use of TENS units showed benefit in patients with improvement in angina or MFR. The other treatments studied, involving statins, calcium-channel blockers, alphablockers, and L-arginine, showed no improvement. However, all of these studies were limited by very small sample sizes (median sample size of 11). A recent study randomized 63 normotensive patients with reported CMD (coronary flow velocity reserve $(\mathrm{CFVR})<2.2$ as assessed by adenosine stress echo) to ramipril vs placebo. They found no difference between the two groups in regards to symptoms or CFVR, suggesting that past studies showing benefit of ACE inhibitors may have been due to antihypertensive effects, with no benefit realized in normotensive patients. ${ }^{16}$ Further randomized prospective trials to evaluate CMD treatment strategies are clearly needed.

Another step in the process of improving our understanding of CMD is the need for routine testing of MFR in patients with INOCA. The differential diagnosis of patients with chest pain and no obstructive epicardial CAD includes both CMD and noncardiac causes, and it is imperative that testing for CMD be done to rule out this entity. Unfortunately, patients without obstructive epicardial CAD are often told their pain is noncardiac; no further work-up is done, resulting in missed opportunities to diagnose and explore treatments for CMD for both symptom management and risk reduction. Alternatively, some clinicians assume patients with chest pain and no obstructive epicardial CAD have CMD without making a formal diagnosis. They start these patients on antianginal treatments that are unnecessary in many instances given that many of these patients will have noncardiac chest pain. In order to determine effective therapies for CMD, it is critical that a formal diagnosis be made, either noninvasively with PET/CMR, or using invasive techniques during coronary angiography. A clinical trial is currently underway that will examine the use of coronary function testing during invasive coronary angiography for patients with angina and no obstructive epicardial CAD. The study cohort will be randomized to assessment of coronary function and subsequent treatment or standard care without this testing. In the coronary function diagnosis/treatment group, MFR will be measured invasively during coronary angiography. If a diagnosis of CMD is made, these patients will be started on an antianginal regimen. This study will provide the first randomized assessment of whether routine testing for CMD translates into clinical benefits. $^{17}$

In summary, the study by Monroy-Gonzalez et al. is significant for demonstrating the long-term prognostic implications for patients with CMD and the confirmed absence of obstructive epicardial CAD. These results further indicate the need to fill the research gap with larger prognostic studies looking at long-term outcomes, assessing whether routine testing for CMD affects outcomes, and evaluating CMD treatments.

\section{Disclosure}

Dr. Schumann has nothing to disclose. Dr. Bourque receives research grant support from Astellas.

\section{References}

1. Bairey Merz CN, Pepine CJ, Walsh MN, Fleg JL. Ischemia and no obstructive coronary artery disease (INOCA). Circulation 2017;135:1075-92.

2. Jespersen L, Hvelplund A, Abildstrom SZ, et al. Stable angina pectoris with no obstructive coronary artery disease is associated with increased risks of major adverse cardiovascular events. Eur Heart J 2012;33:734-44.

3. Camici PG, Crea F. Coronary microvascular dysfunction. N Engl J Med 2007:356:830-40.

4. Kaufmann PA, Camici PG. Myocardial blood flow measurement by PET: Technical aspects and clinical applications. J Nucl Med 2005;46:75-88.

5. Campisi R, Marengo FD. Coronary microvascular dysfunction in women with nonobstructive ischemic heart disease as assessed by positron emission tomography. Cardiovasc Diagn Ther 2017;7:196-205.

6. Camici PG, Rimoldi OE. The clinical value of myocardial blood flow measurement. J Nucl Med 2009;50:1076-87.

7. Farhad H, Dunet V, Bachelard K, Allenbach G, Kaufmann PA, Prior JO. Added prognostic value of myocardial blood flow quantitation in rubidium-82 positron emission tomography imaging. Eur Hear J Cardiovasc Imaging 2013;14:1203-10.

8. Fukushima K, Javadi MS, Higuchi T, et al. Prediction of shortterm cardiovascular events using quantification of global myocardial flow reserve in patients referred for clinical 82Rb PET perfusion imaging. J Nucl Med 2011;52:726-32. 
9. Herzog BA, Husmann L, Valenta I, et al. Long-term prognostic value of $13 \mathrm{~N}$-ammonia myocardial perfusion positron emission tomography. J Am Coll Cardiol 2009;54:150-6.

10. Murthy VL, Naya M, Taqueti VR, et al. Effects of sex on coronary microvascular dysfunction and cardiac outcomes. Circulation 2014;129:2518-27.

11. Tio RA, Dabeshlim A, Siebelink H-MJ, et al. Comparison between the prognostic value of left ventricular function and myocardial perfusion reserve in patients with ischemic heart disease. J Nucl Med 2009;50:214-9.

12. Ziadi MC, deKemp RA, Williams KA, et al. Impaired myocardial flow reserve on Rubidium-82 positron emission tomography imaging predicts adverse outcomes in patients assessed for myocardial ischemia. J Am Coll Cardiol 2011;58:740-8.

13. Löffler AI, Bourque JM. Coronary microvascular dysfunction, microvascular angina, and management. Curr Cardiol Rep 2016;18:1.
14. Brainin P, Frestad D, Prescott E. The prognostic value of coronary endothelial and microvascular dysfunction in subjects with normal or non-obstructive coronary artery disease: A systematic review and meta-analysis. Int J Cardiol 2018;254:1-9.

15. Marinescu MA, Löffler AI, Ouellette M, Smith L, Kramer CM, Bourque JM. Coronary microvascular dysfunction, microvascular angina, and treatment strategies. JACC Cardiovasc Imaging 2015;8:210-20.

16. Michelsen MM, Rask AB, Suhrs E, Raft KF, Høst N, Prescott E. Effect of ACE-inhibition on coronary microvascular function and symptoms in normotensive women with microvascular angina: A randomized placebo-controlled trial. PLoS ONE 2018;13:e0196962.

17. Ford TJ, Corcoran D, Oldroyd KG, et al. Rationale and design of the British Heart Foundation (BHF) Coronary Microvascular Angina (CorMicA) stratified medicine clinical trial. Am Heart $\mathbf{J}$ 2018;201:86-94. 